\title{
Special issue on multi-modal information learning and analytics on big data
}

\author{
Xiaomeng $\mathrm{Ma}^{1} \cdot$ Yan $\mathrm{Sun}^{2}$
}

Received: 24 July 2021 / Accepted: 24 July 2021 / Published online: 4 August 2021

(C) The Author(s), under exclusive licence to Springer-Verlag London Ltd., part of Springer Nature 2021

The world of big data exhibits a rich and complex set of cross-media contents, such as text, image, video, audio and graphics. Thus far, great research efforts have been separately dedicated to big data processing and cross-media mining, with well theoretical underpinnings and great practical success. However, studies jointly considering cross-media big data analytics are relatively sparse. This research gap needs our more attention, since it will benefit lots of real-world applications. Despite its significance and value, it is non-trivial to analyze cross-media big data due to their heterogeneity, large-scale volume, increasing size, unstructured, correlations, and noise. Multi-modal information learning, which can be treated as the most significant breakthrough in the past 10 years, has greatly affected the methodology of computer vision and achieved terrific progress in both academy and industry. From then on, deep learning has been adopted in all kinds of computer vision applications and many breakthroughs have achieved in sub-areas, like DeepFace on LFW competition for face verification and GoogleNet for ImageNet Competition for object categorization. It can be expected that more and more computer vision applications will benefit from multimodal information learning.

This special issue focuses on learning methods to achieve high performance multi-modal information analysis and understanding under uncontrolled environments in large scale, which is also a very challenging problem. Moreover, it attracts much attention from both the academia and the industry. We hope this topic will aggregate top level works on the new advances in multi-modal information from cross-media data. The purpose of this SI is to

Xiaomeng Ma

xiaomengma@szu.edu.cn

1 College of Economics, Shenzhen University, 3688 Nanhai Ave, Nanshan Qu, Shenzhen 518060, China

2 Shanghai University, 99 Shangda Road, Shanghai 200444 , China provide a forum for researchers and practitioners to exchange ideas and progress in related areas. Totally, 20 papers are suggested to EiC for acceptance.

Zhang et al. [1] proposed a method to identify the lithology of drilled formation by using element and gamma spectrum measurement and establishes a reasoning model of intelligent identification of formation lithology by using improved fuzzy clustering algorithm-SVM (IFCM-SVM) method. Liang and Liu [2] establish a safety risk warning system for the construction of underground engineering and to solve the problems existing in current construction of underground engineering, such as large investment, long construction period, complex construction environment, and many unforeseen risk factors. Fang et al. [3] proposed an agricultural entity relationship joint extraction model BERT-BILSTM-LSTM which is applied to the standard data set NYT and self-built agricultural data set AgriRelation. Fan and Zhang [4] study the multi-system fusion of deep neural network and its application in intelligent manufacturing industry. Ji et al. [5] integrated moving average model (ARIMA) is used to predict the short-term price. Hu et al. [6] proposed two methods to optimize the feature representation in article error correction. In order to resolve the issues with overlapping elements in locationrelated applications, an element adjustment method is proposed by Chen and Li [7]. Zhu and Zhang [8] used the special functions of GIS to collect, manage, and analyze data to propose a method of flood disaster risk assessment based on GIS. Li et al. [9] expounded the design of early warning index system, early warning model, establishment of early warning system, and other issues in the macroeconomic early warning theoretical system. Sun et al. [10] explored the application of neural network combined with $\mathrm{X}$-ray and ultrasound in breast examination.

Wang and Ma [11] launched a research on the intelligent Internet of Things traffic management system, with the detection and recognition of stationary vehicles and seat belts as the key analysis targets. Zhang et al. [12] proposed a combination of a neural network-based sports data 
analysis model and a density peak clustering algorithm for unsupervised dimensionality reduction of high-dimensional data. A jujube sorting model in small data sets based on convolutional neural network and transfer learning is proposed by Zheng et al. [13] to meet the actual demand of jujube defects detection. Liu [14] constructs aerobics posture recognition model combined with sensor network. Chen et al. [15] used IACO-BP algorithm to forecast the demand of emergency supplies in the case of flood disaster. Xiong [16] constructs an English network learning interactive evaluation model, studies the influence of the flattening layer in the convolutional neural network structure on the structural complexity, and then proposes spatial pyramid weighted average pooling to optimize the traditional flattened layer. A CDR model named CDCFLFA is presented by Sun et al. [17] to solve the sparsity problem. Xiao and Zhao [18] simulated a perceptive network with 100 nodes deployed randomly. Huang et al. [19] constructed a classification model of clinical burn thermal images based on machine learning algorithms. Gao et al. [20] proposed a multi-granularity feature extraction (MGFE) method based on the gray-level co-occurrence matrix (GLCM) and random forest (RF).

Acknowledgements The guest editors would like to thank Prof. John MacIntyre who is the editor in chief of Neural Computing and Applications. The guest editors would like to thank the reviewers for their high-quality reviews, which provided insightful and constructive feedback to the authors of the papers. The guest editors also would like to thank journal editor Rachel Moriarty, Deepika Sureshkumar, and Rashmi Jenna for their help on submission and publication.

\section{References}

1. Zhang H, Chen Q, Ni P et al (2021) Study on the intelligent identification method of formation lithology by element and gamma spectrum. Neural Comput Appl. https://doi.org/10.1007/ s00521-021-05714-3

2. Liang Y, Liu Q (2021) Early warning and real-time control of construction safety risk of underground engineering based on building information modeling and internet of things. Neural Comput Appl. https://doi.org/10.1007/s00521-021-05755-8

3. Qiao B, Zou Z, Huang Y et al (2021) A joint model for entity and relation extraction based on BERT. Neural Comput Appl. https:// doi.org/10.1007/s00521-021-05815-Z

4. Fan L, Zhang L (2021) Multi-system fusion based on deep neural network and cloud edge computing and its application in intelligent manufacturing. Neural Comput Appl. https://doi.org/10. 1007/s00521-021-05735-y
5. Dou ZW, Ji MX, Wang M et al (2021) Price Prediction of Pu'er tea based on ARIMA and BP Models. Neural Comput Appl. https://doi.org/10.1007/s00521-021-05827-9

6. Hu L, Tang Y, Wu X et al (2021) Considering optimization of English grammar error correction based on neural network. Neural Comput Appl. https://doi.org/10.1007/s00521-020-055912

7. Chen H, Li H (2021) Fast overlap removal for location-related representation elements. Neural Comput Appl. https://doi.org/10. 1007/s00521-021-05825-x

8. Zhu Z, Zhang Y (2021) Flood disaster risk assessment based on random forest algorithm. Neural Comput Appl. https://doi.org/10. 1007/s00521-021-05757-6

9. Yin X, Li J, Huang S (2021) The improved genetic and BP hybrid algorithm and neural network economic early warning system. Neural Comput Appl. https://doi.org/10.1007/s00521-021-057125

10. Song J, Zhang Y, Wang S et al (2021) Neural network combining $\mathrm{X}$-ray and ultrasound in breast examination. Neural Comput Appl. https://doi.org/10.1007/s00521-021-05882-2

11. Wang Z, Ma Y (2021) Detection and recognition of stationary vehicles and seat belts in intelligent Internet of Things traffic management system. Neural Comput Appl. https://doi.org/10. 1007/s00521-021-05870-6

12. Zhang Y, Hou X, Xu S (2021) Neural network in sports cluster analysis. Neural Comput Appl. https://doi.org/10.1007/s00521020-05585-0

13. Ju J, Zheng H, Xu X et al (2021) Classification of jujube defects in small data sets based on transfer learning. Neural Comput Appl. https://doi.org/10.1007/s00521-021-05715-2

14. Liu Q (2021) Aerobics posture recognition based on neural network and sensors. Neural Comput Appl. https://doi.org/10.1007/ s00521-020-05632-w

15. Chen F, Chen J, Liu J (2021) Forecast of flood disaster emergency material demand based on IACO-BP algorithm. Neural Comput Appl. https://doi.org/10.1007/s00521-021-05883-1

16. Hong X (2021) Interactivity of English online learning based on neural network. Neural Comput Appl. https://doi.org/10.1007/ s00521-021-05701-8

17. Yu X, Hu Q, Li H et al (2021) Cross-domain recommendation based on latent factor alignment. Neural Comput Appl. https:// doi.org/10.1007/s00521-021-05737-w

18. Xiao X, Zhao M (2021) Routing optimization strategy of IoT awareness layer based on improved cat swarm algorithm. Neural Comput Appl. https://doi.org/10.1007/s00521-020-05590-3

19. Wu X, Huang W, Wu X et al (2021) Classification of thermal image of clinical burn based on incremental reinforcement learning. Neural Comput Appl. https://doi.org/10.1007/s00521021-05772-7

20. Gao Z, Hu Q, Xu X (2021) Condition monitoring and life prediction of the turning tool based on extreme learning machine and transfer learning. Neural Comput Appl. https://doi.org/10.1007/ s00521-021-05716-1

Publisher's Note Springer Nature remains neutral with regard to jurisdictional claims in published maps and institutional affiliations. 